\title{
How long does it take for the Kondo effect to develop?
}

\author{
Peter Nordlander \\ Department of Physics and Rice Quantum Institute, Rice University, Houston, Texas 77251-1892 \\ Michael Pustilnik and Yigal Meir \\ Physics Department, Ben Gurion University, Beer Sheva, 84105, Israel \\ Ned S. Wingreen \\ NEC Research Institute, 4 Independence Way, Princeton, NJ 08540 \\ David C. Langreth \\ Department of Physics and Astronomy, Rutgers University, Piscataway, NJ 08854-8019
}

The time-development of the Kondo effect is theoretically investigated by studying a quantum dot suddenly shifted into the Kondo regime by a change of voltage on a nearby gate. Using time-dependent versions of both the Anderson and Kondo Hamiltonians, it is shown that after a time $t$ following the voltage shift, the form of the Kondo resonance matches the timeindependent resonance at an effective temperature $T_{\text {eff }}=T / \tanh (\pi T t / 2)$. Relevance of the buildup of the Kondo resonance to the transport current through a quantum dot is demonstrated.

PACS numbers: 72.15.Qm, 85.30.Vw, 73.50.Mx

The Kondo effect in quantum dots has been observed in several recent experiments [1]. Beyond verifying theoretical predictions [2,3], these experiments demonstrate that quantum dots can serve as an important new tool to study strongly correlated electron systems. Unlike magnetic impurities in metals, the physical parameters of the quantum dot can be varied continuously, which allows, for example, systematic experimental study of the crossover between the Kondo, the mixed-valence, and the non-Kondo regimes. Moreover, the quantum dot system opens the possibility of directly observing the timedependent response of a Kondo system, as there is a well developed technology for applying time-dependent perturbations to dots 4 . Along these lines, several theoretical works have addressed the behavior of a Kondo impurity subject to ac driving [5]. However, a clearer picture of the temporal development of many-body correlations is obtained if the impurity is subject to a sudden shift in energy. Specifically, by applying a step-like impulse to a nearby gate, the dot can be suddenly shifted into the Kondo regime, and the buildup of the correlated state observed in the transport current.

In this Letter, we analyze the behavior of a quantum dot following a sudden shift into the Kondo regime. The time-dependent spectral function is evaluated within the non-crossing approximation (NCA) 3, 6, 8], as is the transport current in response to a pulse train. The latter provides an experimental window on the development of the Kondo resonance. Employing the Kondo Hamiltonian, we show that a finite development time $t$ is perturbatively equivalent to an increase in the effective tem- perature.

We treat a quantum dot coupled by tunnel barriers to two leads (inset to Fig. 2). Only one spin-degenerate level on the dot is considered, which is a good approximation at low temperatures. A time-dependent voltage $V_{g}(t)$ is applied to a nearby gate, causing a proportionate shift in the energy of the level $\epsilon_{\mathrm{dot}}(t)$. If the Coulomb interaction between electrons prevents double occupancy of the dot, the system is described by the $U=\infty$ Anderson Hamiltonian for a magnetic impurity,

$$
\sum_{\sigma} \epsilon_{\mathrm{dot}}(t) n_{\sigma}+\sum_{k \sigma}\left[\epsilon_{k \sigma} n_{k \sigma}+\left(V_{k} c_{k \sigma}^{\dagger} c_{\sigma}+\text { H.c. }\right)\right],
$$

with the constraint that the occupation of the dot cannot exceed one electron. Here $c_{\sigma}^{\dagger}$ creates an electron of spin $\sigma$ in the quantum dot, with $n_{\sigma}$ the corresponding number operator; $c_{k \sigma}^{\dagger}$ creates an electron in the leads, with $k$ representing all quantum numbers other than spin, including the labels, left and right, for the leads. $V_{k}$ is the tunneling matrix element through the appropriate barrier. The quantum dot is occupied by a single electron provided the level energy $\epsilon_{\text {dot }}$ lies at least a resonance width $\Gamma_{\text {dot }}$ [9] below the chemical potential of the leads. At low temperatures, the resulting free spin on the dot forms a singlet with a spin drawn from the electrons in the leads - this is the Kondo effect. The Kondo temperature, beneath which the strongly correlated state is established, is given by $T_{K} \simeq D^{\prime} \exp \left(-\pi\left|\epsilon_{\text {dot }}\right| / \Gamma_{\text {dot }}\right)$, where $D^{\prime}$ is a high energy cutoff [10]. The signature of this correlated state is a peak at the Fermi energy in the spectral density of the dot electrons. This peak, in turn, dramatically enhances transport through the dot, allowing perfect transmission 


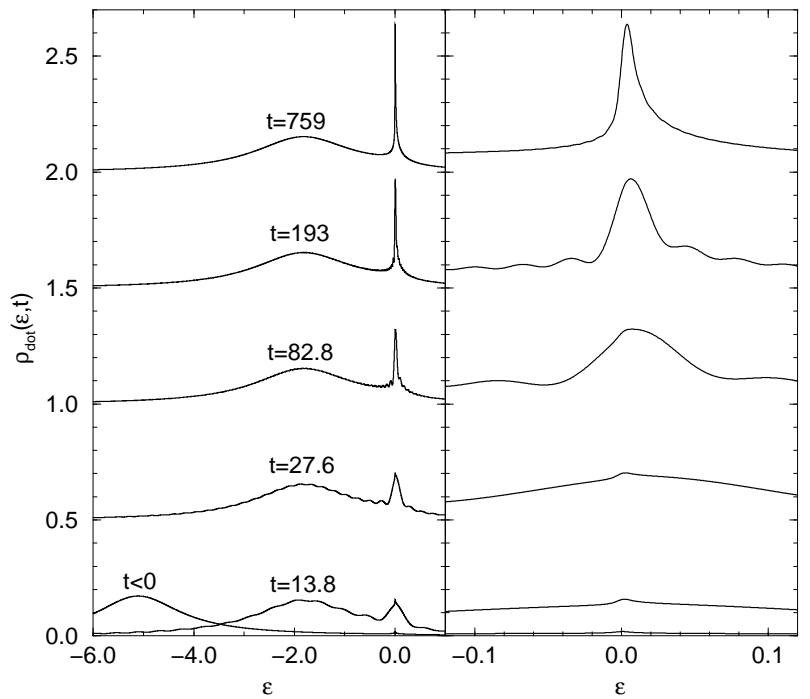

FIG. 1. Spectral density $\rho_{\operatorname{dot}}(\epsilon, t)$ vs. energy $\epsilon$ at various times following a step-function change in the level energy $\epsilon_{\mathrm{dot}}(t)=-5+3 \theta(t)$. The ordinates for positive times are successively offset by 0.5 units. For $t<0, \rho_{\text {dot }}(\epsilon, t)$ is identical to the equilibrium spectral density at $\epsilon_{\text {dot }}=-5$ while for the largest time shown it is indistinguishable on this scale from the equilibrium spectral density at $\epsilon_{\mathrm{dot}}=-2$. Throughout this work energies are given in units of $\Gamma_{\text {dot }}$, and times in units of $1 / \Gamma_{\text {dot }}$, with $\hbar=1$. Here $T=0.0025$.

at zero temperature [2].

We employ the non-crossing approximation (NCA) to analyze the spectral density and transport through the dot in the presence of a time-dependent level energy $\epsilon_{\text {dot }}(t)$. The NCA is based on an exact transformation of the $U=\infty$ Anderson model in Eq. (11) into a slaveboson Hamiltonian [6]. The latter is then solved selfconsistently to second order in the tunneling matrix elements $V_{k}$. The NCA approximation gives reliable results for temperatures down to $T<T_{K}$, and its timedependent formulation has been discussed at length in previous works [7, 8 . We define a time-dependent spectral density for the dot electrons as 11

$$
\rho_{\mathrm{dot}}(\epsilon, t) \equiv \operatorname{Re} \int_{0}^{\infty} \frac{d \tau}{\pi} e^{i \epsilon \tau}\left\langle\left\{c_{\sigma}(t), c_{\sigma}^{\dagger}(t-\tau)\right\}\right\rangle .
$$

The spectral density is the same for both spins in the absence of a magnetic field. In equilibrium, $\rho_{\text {dot }}(\epsilon, t)$ is independent of $t$ and reduces to the usual density of states. Furthermore, $\rho_{\text {dot }}(\epsilon, t)$ is causal, i.e. it depends only on the behavior of the level energy for times earlier than $t$. In Fig. (1), we have plotted the time-dependent spectral density for several times following an abrupt shift of the level energy 12]. Before the shift, the level energy is so low, $\epsilon_{\mathrm{dot}}=-5$, that the Kondo temperature is much smaller than the physical temperature, and so there is no noticeable Kondo peak in the spectral density. At $t=0$, the level energy is abruptly shifted to $\epsilon_{\mathrm{dot}}=-2$, giving $T_{K} \sim 10^{-3}$, comparable to $\mathrm{T}$. The Kondo peak

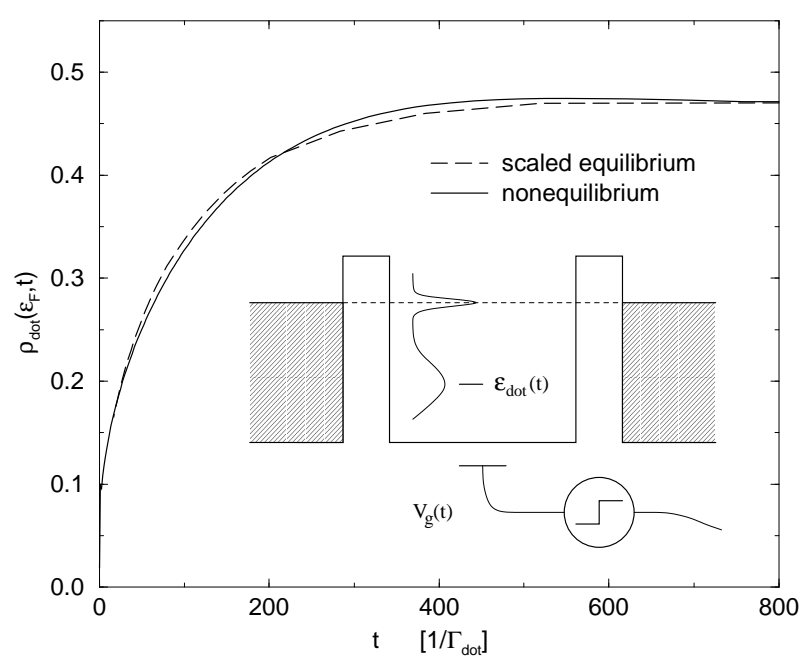

FIG. 2. Solid curve: time-dependent spectral density $\rho_{\text {dot }}\left(\epsilon_{F}, t\right)$ at the Fermi energy vs. time $t$ following the same step-function change in the level energy used in Fig. 1. The temperature is $T=0.0025$. Dashed curve: equilibrium spectral density $\rho_{\text {dot }}\left(\epsilon_{F}\right)$ at the Fermi energy with temperature set according to Eq. (7): $T_{\text {eff }}(t)=T / \tanh (\pi T t / 2)$, with $T=0.0025$. Inset: schematic of the quantum-dot single electron transistor.

thereafter grows with a characteristic time dependence shown in Fig. (2), approaching a new equilibrium value at long times $t \sim 1 / T_{K}$.

To develop an analytical theory for the time development of the Kondo resonance, we consider the timedependent Kondo Hamiltonian,

$$
\begin{gathered}
H_{K}=\sum_{k \sigma} \epsilon_{k \sigma} n_{k \sigma}+J(t) \mathbf{S} \cdot \mathbf{s} \\
\mathbf{s}=\sum_{\alpha \alpha^{\prime}} \psi_{\alpha}^{\dagger} \frac{\sigma_{\alpha \alpha^{\prime}}}{2} \psi_{\alpha^{\prime}}, \mathbf{S}=\sum_{\beta \beta^{\prime}} c_{\beta}^{\dagger} \frac{\sigma_{\beta \beta^{\prime}}}{2} c_{\beta^{\prime}}, n_{c}=\sum_{\beta} c_{\beta}^{\dagger} c_{\beta} .
\end{gathered}
$$

Here $\psi_{\alpha}^{\dagger}$ creates a conduction-band electron at the site of the Kondo impurity, and the $\sigma$ are the Pauli spin matrices. For near Fermi surface properties, the Anderson Hamiltonian reduces to the simpler Kondo Hamiltonian with $J(t)=2\left|V_{k_{F}}^{2} / \epsilon_{\text {dot }}(t)\right|$ when the site is occupied by a single electron 13]. For the case of interest, in which the level energy $\epsilon_{\mathrm{dot}}$ is suddenly shifted into the Kondo regime at $t=0$, it is adequate to consider a sudden switching on of the Kondo coupling, $J(t)=J \theta(t)$. We have written the Kondo Hamiltonian in terms of Abrikosov's pseudofermion representation [14], where $c_{\beta}^{\dagger}$ creates a pseudofermion of spin $\beta$, which represents the magnetic impurity. In order to restrict the states to the physical subspace, we have to impose a constraint $n_{c}=1$. This can be done by adding a term $\lambda n_{c}$ to the Hamiltonian [14] and taking the limit $\lambda \rightarrow \infty$. Since we are interested in a sudden switching on of the Kondo coupling $J \mathbf{S} \cdot \mathbf{s}$, we are able to implement the constraint $n_{c}=1$ by explicitly creating a pseudofermion at $t=0$. Then, as the 

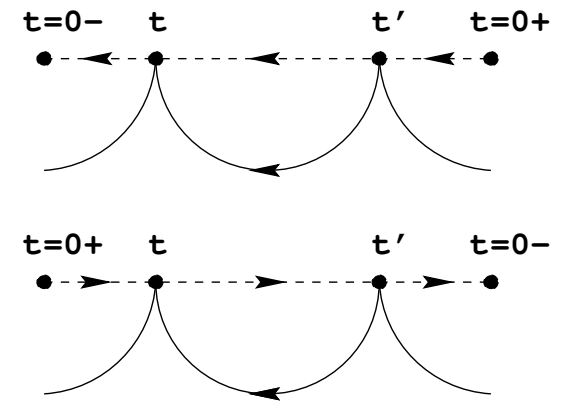

FIG. 3. Contributions of order $J^{2}$ to the renormalized conduction electron scattering vertex, from the Kondo Hamiltonian in Eq. (3). Solid lines are conduction electron propagators and dashed lines are pseudofermion propagators. Summation over internal spins is implied.

pseudofermion number is conserved by $H_{K}+\lambda n_{c}$, and we have $n_{c}=0$ for $t<0$ because of the large pseudofermion energy $\lambda \rightarrow \infty$, we obtain an abrupt turn on of the Kondo coupling at $t=0$ and all later expectations are taken in the physical subspace $n_{c}=1$.

The analytical signature of the Kondo effect is the logarithmic divergence of perturbation theory in the dimensionless coupling $J \rho$, where $\rho$ is the density of conduction electron states per spin direction at the Fermi level. Indeed, for $T<T_{K}$ perturbation theory in $J \rho$ fails, even for small $J \rho$. For $T>T_{K}$, temperature cuts off the logarithmic divergences and perturbation theory is reliable [14. We find that a finite time $t$ following a sudden switching on of the Kondo coupling also results in a convergent perturbation theory. To demonstrate this, we focus on the simplest quantity that diverges in perturbation theory. Specifically, we calculate the scattering vertex $\gamma^{p p}\left(t, t^{\prime}\right)$ to order $J^{2}$. Physically, this quantity represents the lowest order change in $J$ due to multiple scattering from the Kondo impurity. Since abruptly turning on the Kondo coupling creates a nonequilibrium state of the system, we use Keldysh Green functions with $p= \pm 1$ for the outward/backward branches. In time, the Keldysh contour runs from $-\infty$ to $\infty(p=+1)$ and then from $\infty$ to $-\infty$ $(p=-1)$. As shown in Fig. (3), there are two contributions at order $J^{2}$, one with the conduction electron line and the pseudofermion line parallel and one with the lines antiparallel. Evaluating the diagrams in Fig. (3), and keeping only logarithmically divergent contributions in addition to the bare vertex, we find

$$
\begin{aligned}
\gamma^{p p^{\prime}}\left(t, t^{\prime}\right)= & p \delta_{p p^{\prime}} \frac{J}{4}\left(\sigma_{\alpha \alpha^{\prime}} \sigma_{\beta \beta^{\prime}}\right) \theta(t) \theta\left(t^{\prime}\right) \\
& \times\left[1-\frac{J}{2} G_{0}^{p p}\left(t-t^{\prime}\right) \operatorname{sgn}\left(t-t^{\prime}\right)\right] .
\end{aligned}
$$

(Note that in this order there is no logarithmic contribution that is off-diagonal in the Keldysh indices.) Here $G_{0}^{p p}\left(t-t^{\prime}\right)$ is the bare time-ordered (for $p=+1$ ) or antitime-ordered (for $p=-1$ ) Green function for conduction electrons at the site of the Kondo impurity. For $\left|t-t^{\prime}\right| \gg 1 / D(D$ is a high-energy cutoff $)$ it takes the form 15]

$$
G_{0}^{p p}\left(t-t^{\prime}\right) \rightarrow \frac{-\pi \rho T}{\sinh \left[\pi T\left(t-t^{\prime}\right)\right]}
$$

Fourier transforming (画) with respect to the time difference $t-t^{\prime}$, and taking the limit of zero frequency to obtain the effective scattering vertex at time $t$ for electrons near the Fermi energy, we find

$$
\gamma(t, \omega \rightarrow 0) \propto J\left\{1+\frac{1}{2} \rho J \ln \left[\frac{D}{T} \tanh \left(\frac{\pi T t}{2}\right)\right]\right\} .
$$

For $T t \gg 1$ this reduces to the usual equilibrium form, $\gamma \propto J\left[1+\frac{1}{2} \rho J \ln \frac{D}{T}\right]$, with the logarithmic divergence cut off only by temperature. However, since in our case the Kondo coupling exists only for times $t>0$, the result contains an additional cutoff due to the finite time allowed for spin-flip scattering. Formally, the finite time $t$ since the onset of the Kondo coupling can be absorbed into an increase in the effective temperature,

$$
T_{\text {eff }}=\frac{T}{\tanh (\pi T t / 2)} \text {. }
$$

How accurately does this effective temperature represent the time development of the Kondo resonance at the Fermi surface? To test the applicability of Eq. (7) beyond perturbation theory, we have compared the timedependent NCA results to time-independent equilibrium NCA results at the corresponding effective temperature. The agreement, with no free parameters, is quite good as is seen in Fig. (2). Note that at short times $T_{\text {eff }} \simeq 2 / \pi t$. Hence, the buildup of the Kondo resonance is governed by a type of energy-time uncertainty relation: after a time $t$ the Kondo resonance is cut off by an energy $\sim 1 / t$ [16]. Thus we expect saturation of the Kondo peak at a time $t \sim 1 / T_{K}$, as indeed is observed numerically.

Finally, we consider how the buildup of the Kondo resonance can be observed experimentally. In steady state, the linear-response conductance $G$ through a dot symmetrically coupled to its leads is given by [17]

$$
G=\frac{e^{2}}{\hbar} \frac{\Gamma_{\mathrm{dot}}}{2} \int d \epsilon \rho_{\mathrm{dot}}(\epsilon)\left(-\frac{\partial f(\epsilon)}{\partial \epsilon}\right),
$$

where $f(\epsilon)$ is the Fermi function, and $\hbar$ is explicitly included for clarity. If a periodic gate voltage is applied to the dot, formula (8) is still valid if $G$ is replaced by the time-averaged conductance $\langle G\rangle$, and $\rho_{\operatorname{dot}}(\epsilon)$ is replaced by the average of the time-dependent spectral density $\left\langle\rho_{\mathrm{dot}}(\epsilon, t)\right\rangle$. Consider a periodic signal consisting of an "on" pulse of duration $\tau_{\text {on }}$ which brings the dot into the Kondo regime followed by an "off" pulse which moves it back out of the Kondo regime. During each on pulse, $\rho_{\text {dot }}\left(\epsilon_{F}, t\right)$ will build up to a maximum at time $\tau_{\text {on }}$ and then rapidly decrease back to a low value during the off pulse. The differential increase of conductance as the duration of the on 


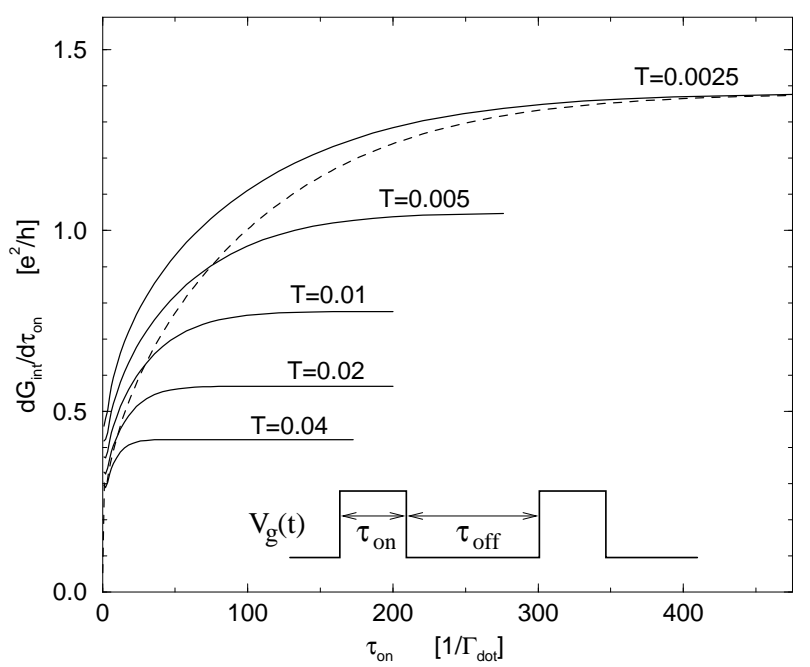

FIG. 4. Solid curves: derivative of $G_{\text {int }}$ (in units of $e^{2} / h$ ) with respect to duration $\tau_{\text {on }}$ of "on" gate-voltage pulses, at various temperatures. $G_{\text {int }}$ is the conductance integrated over a full cycle of gate voltage. Dashed curve: $-\pi \int d \epsilon \Gamma_{\operatorname{dot}} f^{\prime}(\epsilon) \rho_{\operatorname{dot}}\left(\epsilon, t=\tau_{\text {on }}\right)$ for $T=0.0025$. Inset: schematic periodic gate-voltage pulse train. The level energy is $\epsilon_{\mathrm{dot}}=-2$ in the on state and $\epsilon_{\mathrm{dot}}=-5$ in the off state. The duration of the off period, $\tau_{\text {off }}$ is long enough to allow transients from each on pulse to die out.

pulse is increased will therefore reflect the magnitude of the spectral density near or at the Fermi energy at a time $\tau_{\text {on }}$ following the shift into the Kondo regime. In Fig. (4), we have plotted the differential with respect to $\tau_{\text {on }}$ of the conductance, with a fixed off-pulse duration $\tau_{\text {off }}$. The conductance is integrated over the period, rather than time-averaged, to remove effects due to the changing duration of the period, i.e. $G_{\text {int }}=\left(\tau_{\text {on }}+\tau_{\text {off }}\right)\langle G\rangle$. This measurable transport quantity provides a probe of the time-development of the Kondo resonance [18].

In conclusion, we have analyzed the response of a quantum dot to a sudden shift of gate voltage which takes the dot into the regime of the Kondo effect. The buildup of many-body correlations between the dot and the leads follows an uncertainty principle: at time $t$ the Kondo resonance is cut off by an energy $\sim 1 / t$. Within perturbation theory in the Kondo coupling, we find that the finite time $t$ plays the role of an increased effective temperature $T_{\text {eff }}=T / \tanh (\pi T t / 2)$. To experimentally probe the buildup of the Kondo resonance, we propose applying a train of square gate-voltage pulses to the dot. The derivative of current with respect to duration of the "on" pulse accurately reproduces the time-dependent amplitude of the Kondo resonance.

The work was supported in part by NSF grants DMR 95-21444 (Rice) and DMR 97-08499 (Rutgers). Work at BGU was supported by the The Israel Science Foundation - Centers of Excellence Program. One of us (MP) acknowledges the support of a Kreitman Fellowship.
[1] D. Goldhaber-Gordon et al., Nature 391, 156 (1998); D. Goldhaber-Gordon et al., Phys. Rev. Lett. 81, 5225 (1998); S. M. Cronenwett, T. H. Oosterkamp, and L. P. Kouwenhoven, Science 281, 540 (1998); F. Simmelm et al., cond-mat/9812153. T. Schmidt et al., unpublished.

[2] L. I. Glazman and M. E. Raikh, Pis'ma Zh. Eksp. Teor. Fiz. 47, 378 (1988) [JETP Lett. 47, 452 (1988)]; T. K. $\mathrm{Ng}$ and P. A. Lee, Phys. Rev. Lett. 61, 1768 (1988); S. Hershfield, J. H. Davies, and J. W. Wilkins, Phys. Rev. Lett. 67, 3720 (1991).

[3] Y. Meir, N. S. Wingreen, and P. A. Lee, Phys. Rev. Lett. 70, 2601 (1993); N. S. Wingreen and Y. Meir, Phys. Rev. B 49, 11040 (1994).

[4] L. P. Kouwenhoven et al., in Mesoscopic Electron Transport, edited by L. L. Sohn, L. P. Kouwenhoven, and G. Schön (Kluwer, Netherlands, 1997).

[5] A. Schiller and S. Hershfield, Phys. Rev. Lett. 77, 1821 (1996); T. K. Ng, Phys. Rev. Lett. 76, 487 (1996); Y. Goldin and Y. Avishai, Phys. Rev. Lett. 81, 5394 (1998).

[6] N. E. Bickers, Rev. Mod. Phys. 59, 845 (1987).

[7] D. C. Langreth and P. Nordlander, Phys. Rev. B 43, 2541 (1991).

[8] H. Shao, D. C. Langreth, and P. Nordlander, Phys. Rev. B 49, 13929 (1994).

[9] We define $\Gamma_{\operatorname{dot}}(\epsilon)=2 \pi \sum_{k}\left|V_{k}\right|^{2} \delta\left(\epsilon-\epsilon_{k}\right)$, a slowly varying quantity. The notation $\Gamma_{\text {dot }}$ with no energy specified will always refer the value at the Fermi level.

[10] For $U=\infty, D^{\prime} \simeq \sqrt{D \Gamma_{\mathrm{dot}} / 4}$, where $2 D$ is the effective bandwidth. The calculations here used a parabolic band of total width $40 \Gamma_{\text {dot }}$.

[11] A.-P. Jauho, N. S. Wingreen, and Y. Meir, Phys. Rev. B 50, 5528 (1994).

[12] Our calculations are based on the approximation that the switching time, $\tau_{s}$, is exactly zero. In reality, $\tau_{s}$ is always a finite time. Our results are valid for finite $\tau_{s}$ as well, provided that $t \gg \tau_{s}$.

[13] J. R. Schrieffer and P. A. Wolff, Phys. Rev. 149, 491 (1966).

[14] A. A. Abrikosov, Physics 2, 5 (1965).

[15] G. Yuval, and P. W. Anderson, Phys. Rev. B 1, 1522 (1970).

[16] By evaluating the conduction electron self-energy to order $J^{3}$, we have directly confirmed the $\sim 1 / t$ cutoff for the Kondo peak in the spectral density.

[17] Y. Meir and N. S. Wingreen, Phys. Rev. Lett. 68, 2512 (1992).

[18] The difference between the dashed and solid curves at small $\tau_{\text {on }}$ reflects the finite decay-time of the Kondo resonance after the pulse is switched off. 